\title{
Design and simulation of current-fed dual-active full-bridge DC/DC converter control system applied to proton exchange membrane fuel cell
}

\author{
Fengjiao Dai*, Xiang Wang, and Wei Kang \\ State Key Laboratory of Advanced Power Transmission Technology (Global Energy Interconnection \\ Research Institute Co., Ltd.), Changping District, Beijing 102209, China
}

\begin{abstract}
Proton exchange membrane fuel cells (PEMFC) have been increasingly applied in clean and efficient distributed power generation systems in recent years. However, the output characteristics of PEMFC are relatively soft due to the influence of multiple polarization overvoltage. With the increasement of current density, the output voltage shows to be a nonlinear downward trend. In addition, in order to ensure the lifetime of PEMFC, the output ripple current is often concerned. Considering above characteristics, in order to achieve efficient power transmission of the PEMFC system, this paper adopts the current-fed dual-active full-bridge (DAB) DC/DC converter topology, which can achieve soft switching of most switches. Furthermore, it can restrain the characteristics of the current ripple. Hence, the power transmission efficiency and current ripple requirements of PEMFC are fulfilled. Finally the current-fed DAB is designed and simulated through PSIM. The C-block is applied to simulate the PEMFC nonlinear power supply according to the actual PEMFC parameters. In order to achieve efficient constant power transmission of the nonlinear PEMFC, a novel power closed-loop control strategy is proposed. Keywords: Current-fed DAB; Proton exchange membrane fuel cell; Soft switch; DC/DC converter.
\end{abstract}

\section{Introduction}

As a new power generation technology, the hydrogen fuel cell energy's conversion is not restricted to Carnot cycle, with features of high energy conversion rate ${ }^{[1]}$, clean without pollution, and zero noise, which provides a new route for the solution of energy crisis and environmental pollution. PEMFC can take full advantage of new energy like industrial byproduct hydrogen and abandoned wind and light to produce hydrogen to achieve decoupling of hydrogen and new energy development and utilization in different time scale, which can effectively achieve demand side management and with important roles to play in areas like improving energy comprehensive utilization level, reducing energy use cost, promoting distributive power connection, guarantee power supply and demand response.

\footnotetext{
* Corresponding author: daifengjiao@geiri.sgcc.com.cn
} 
Due to the soft feature and big fluctuation of the output voltage generated by PEMFC, as well as the slow response of output power and only one-way flow, a converter must be equipped to regulate, control and manage the output of fuel cell before grid connection operation. In the paper, a converter topology is adopted to PEMFC whose voltage change range of $40 \mathrm{~V} \sim 75 \mathrm{~V}$ as input power, which connects to $750 \mathrm{~V}$ DC bus voltage end and transmit at constant power.

Due to the soft output feature of PEMFC, it is easy to be affected by load, and its output power is unstable under no closed loop control, meanwhile in order to ensure PEMFC's life, it needs to adopt a DC/DC converter with high step-up ratio, high efficiency, and small input current ripple wave. Because of its features of achieving high power density, natural zero voltage switch (ZVS) characteristics, high efficiency, symmetrical structure and simple control, a dual-active bridge bidirectional DC/DC converter (Dual-Active-Bridge, $\mathrm{DAB}$ ) is widely used in medium, and large power supplies. However, voltage source DAB has some disadvantages, such as large input ripple current, limited soft switching range, large circulating current through devices and transformers, and low efficiency in high voltage and high current applications. In [2], a current type DAB converter is applied into the photovoltaic system that needs to have high step-up ratio DC/DC converter. In order to increase gain of voltage conversion, the current-fed type DC/DC converter is the best choice $^{[3-6]}$. Current type DAB has been proved to be applied to low voltage and big current field.

In [7], a current-fed type dual-active full-bridge DC/DC converter is proposed, where that converter eliminates the clamp forward circuit and passive buffer device in the circuit by secondary modulation technique, and realizes zero current shutdown (ZCS) and zero voltage switching of the primary side device and zero voltage switching of secondary side device, which significantly reduces switching loss.

In this paper, a PEMFC's structure and external features are provided, and the working principles of that current type DAB topological structure then are analyzed. According to the proposed new power closed loop control strategy, a power closed loop design and simulation modeling of non-linear PEMFC voltage source is achieved, so as to achieve the effective constant power transmission.

\section{PEMFC structure and external features}

\subsection{PEMFC structure}

PEMFC is composed of cathode tank, anode tank and electrolyte membrane (proton exchange membrane). Hydrogen reaches to anode through pipes or gas guide plate. Under the action of anode catalyst, hydrogen molecules dissociate into positively charged hydrogen ions (protons) and release negatively charged electrons. Hydrogen ions travel through the electrolyte (proton exchange membrane) to the cathode, while electrons travel through an external circuit to the cathode. Electrons form an electric current in an external circuit, and with appropriate connections, it can output electrical energy to a load. At the other end of the cell, oxygen (or air) travels to the cathode through a pipe or gas guide plate. Under the action of cathode catalyst, oxygen reacts with hydrogen ions and electrons to produce water, meanwhile releasing heat.

\subsection{PEMFC external features}

Due to the special loss mechanism such as activation overvoltage, ohm overvoltageand concentration overvoltage, in operation, the single PEMFC voltage drops occur as the 
output current changes. Under the medium power level, PEMFC STACK's output voltage composed of multiple PEMFCs generates big change with the load fluctuation. With the increase of PEMFC STACK, the output current increases continuously and maintains at a relatively high level. On the contrary, the output voltage is often relatively low. So as to the application occasions where voltage is low and current is big of PEMFC generation system, the DC/DC converter must be matched. Only in this way, the output voltage can be kept at a relatively high level.

Referring to the output characteristic curve of Nedstack manufacture's model FCS 10XXL's PEMFC, as shown in Fig.1, the output voltage reduces with the current increase, and its reduction scale is big. So this paper combines the current type DAB with high voltage gain for PEMFC, so as to achieve $750 \mathrm{~V}$ DC bus constant power transmission.

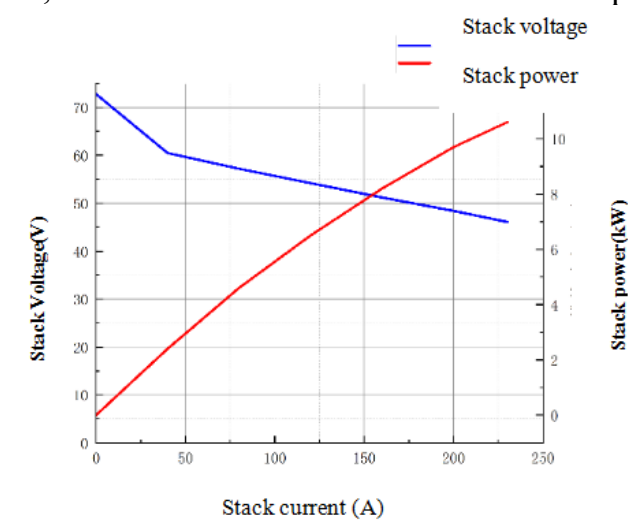

Fig. 1. PEMFC output characteristic curve.

\section{PEMFC current-fed type dual-active full-bridge DC/DC converter}

\subsection{Topological structure and features}

PEMFC current-fed type dual-active full-bridge DC/DC converter (DAB) topology is shown as Fig.2. $D_{0}$ is the reverse blocking diode, which prevents the negative current flows into battery pack and cause damage to PEMFC. CF-DAB is composed of two $\mathrm{H}$ bridges and one high frequency transformer, altogether 8 switch tubes, $S_{I} \sim S_{8}$ respectively. Its inverse parallel diodes are $D_{1} \sim D_{8}$ respectively. The buffer capacitance of each switch tube is $C_{I} \sim C_{8}, L$ is boost inductor, $L_{l k}$ is transformer leakage inductance, and $C_{0}$ is output capacitance.

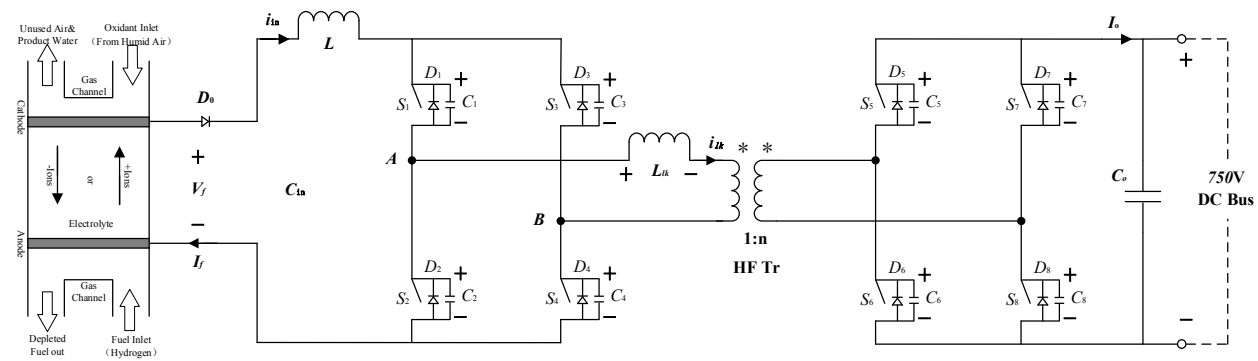

Fig. 2. PEMFC current-fed dual-active full-bridge DC/DC converter. 
By matching of duty cycle of the primary and secondary side switch tube, this converter achieves the zero current shutdown and zero voltage switching of primary side switch tube, and the zero voltage shutdown of the secondary side switch tube, which reduces the switching loss of switch tubes. Due to the voltage reflection of DC power grid, the voltage of the primary side switch tube is clamped at $V_{o} / n$, which eliminates voltage spike. The converter transmission efficiency is greatly improved in this circuit.

\subsection{Operating model analysis}

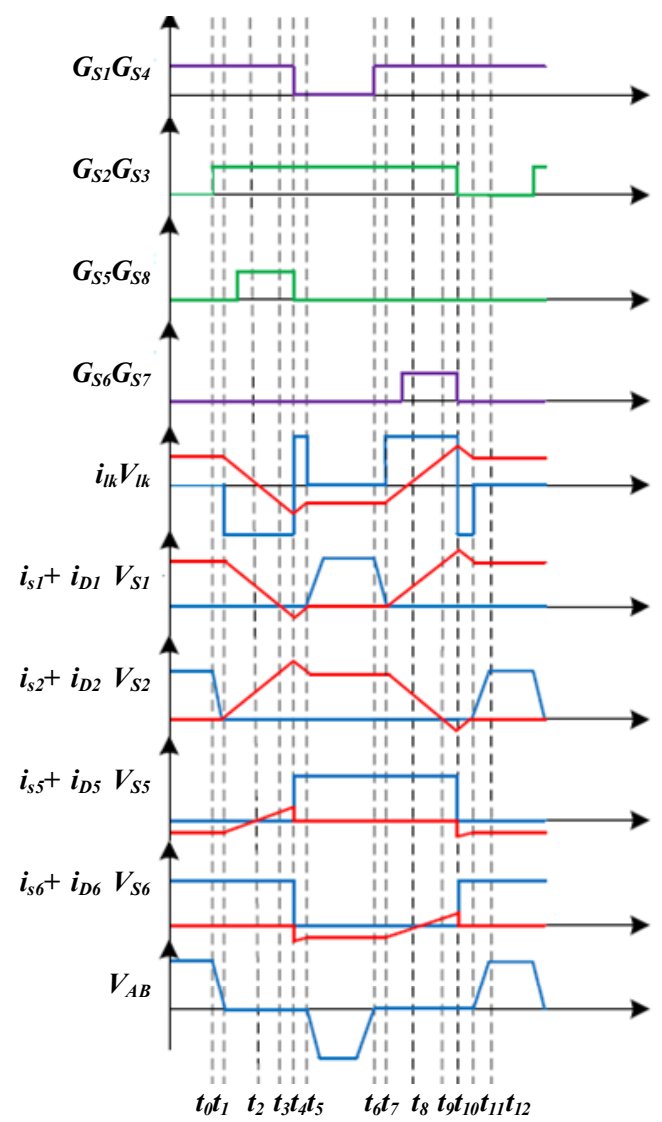

Fig. 3. Working waveform.

Working waveform is shown as Fig.3. Before $t_{0}$, the primary side switch tubes $S_{1}, S_{4}$ and the secondary side diodes $D_{5}, D_{8}$ turn on, the power transmits from primary side to secondary side. $i_{l k}=i_{s 1}=i_{s 4}=i_{i n}, i_{D 5}=i_{D 8}=i_{i n} / n$.

Operating model $1\left[t_{0}, t_{1}\right]$ : In $t_{0}-t_{1}$ period, the switch tubes $S_{2}$ and $S_{3}$ are triggered, and then buffer capacitance $C_{2}$ and $C_{3}$ are discharged. Because of the extremely low buffer capacitance and extremely short time, power transmits from primary side to secondary side. At the end of this period, the voltage of switch tubes $S_{2}, S_{3}$ reduces to zero, and zero voltage start is enforced.

Operating model $2\left[t_{1}, t_{2}\right]$ : In $t_{1}-t_{2}$ period, $S_{2}$ and $S_{3}$ are turned on, all of switch tubes in the same bridge arms of primary side keep conducting state, the voltage of the primary side leakage inductance is $V_{o} / n$, at this time $V_{l k}=V_{o} / n$. The leakage inductance current starts to decrease linearly with slope $k . i_{s 2}$ and $i_{s 3}$ begin to increase from zero with $-k / 2$ slope, $i_{s I}$ and 
$i_{s 4}$ reduce linearly with $k / 2$ slope, power stores on leakage inductance $L_{l k}$ begins to transmit to secondary side. At the end of this period, $S_{5}$ and $S_{8}$ begins are triggered. $i_{l k}=i_{D 5}=i_{D 8}=0$, the slope is $k=V_{o} / n L_{l k}$. Owing to the $D_{5}$ and $D_{8}$ turning on, the voltage of switch tubes $S_{5}$ and $S_{8}$ are clamped to zero before turning on.

Operating model $3\left[t_{2}, t_{3}\right]$ : At the moment of $t=t_{2}$, for the switch tubes $S_{5}$ and $S_{8}, Z$ VS is completed. The voltage reflected from secondary side to the primary side is still $V_{o} / n$, so the leakage inductance current ilk continues to reduce with the same slope $k$. Leakage inductance is to absorb power, and secondary side power feeds back to primary side. $i_{s 2}$ and $i_{s 3}$ continue to increase with $-k / 2$ slope, and the currents of the $i_{s l}$ and $i_{s 4}$ continue to reduce linearly with $k / 2$ slope. At the end of this period, $i_{s I}=i_{s 4}=0, i_{s 2}=i_{s 3}=-i_{i n}$, the two currents $i_{D I}$ and $i_{D 4}$ begin to stream conducting, making $S_{1}$ and $S_{4}$ voltage clamped at zero and maintain in $t_{3}-t_{5}$ period.

Operating model $4\left[t_{3}, t_{4}\right]$ : In $t_{3}-t_{4}$ period, Two switches of $i_{D 1}$ and $i_{D 4}$ are opened continuously, the status of the secondary side switch is unchanged, leakage inductance current $i_{l k}$ continues to reduce with slope $k, i_{s 2}$ and $i_{s 3}$ continue to increase with $-k / 2$ slope, and leakage inductance continues to absorb power. At the end of this period, $S_{5}$ and $S_{8}$ stop to be triggered, $S_{5}$ and $S_{8}$ current transfer to $D_{6}$ and $D_{7}$. The polarity of the reflected voltage on the leakage inductance is opposite, and the absolute value is unchanged. Leakage inductance current $i_{l k}$ begins to change at the opposite direction with slope $-k$, all of the leakage inductance current ilk and $i_{s 2}, i_{s 3}$ reach peak value.

Operating model $5\left[t_{4}, t_{5}\right]$ : In $t_{4}-t_{5}$ period, $D_{6}$ and $D_{7}$ stream conduct, the polarity of the reflected voltage on the leakage inductance is opposite, and the absolute value is unchanged. Leakage inductance current $i_{l k}$ begins to increase from peak value with slope $-k$, the two currents of $i_{s 2}$ and $i_{s 3}$ begin to reduce from peak value with slope $-k / 2$, and leakage inductance releases power to the secondary side. At the end of this period, $i_{l k}=-i_{i n}, i_{D 6}=i_{D 7}=-$ $i_{i n} / n, i_{s 2}=i_{s 3}=i_{i n}, i_{D 1}=i_{D 4}=0$. Buffer resistance $C_{1}$ and $C_{4}$ begin to charge, switch tubes $S_{1}$ and $S_{4}$ achieve ZCS, which reduce turn-off loss.

Operating model $6\left[t_{5}, t_{6}\right]$ : In $t_{5}-t_{6}$ period, the power is transferred from primary side to secondary side, the fuel cell charges to buffer capacitance $C_{l}$ and $C_{4}$ and its value is $V_{o} / n$.

Operating model $7\left[t_{6}, t_{7}\right]$ : In $t_{6}-t_{7}$ period, the converter achieves a convert of fuel cell output current from switch tubes $S_{1}, S_{4}$ to switch tubes $S_{2}, S_{3}$, the circuit enters a stable condition, the power is transferred from primary side to secondary side, $i_{l k}=i_{s 2}=i_{s 3}=-i_{i n}$. It can be known from the analysis process from operating model 1 to operating model 6 , in which this switch process realizes soft switch and eliminates voltage spike.

Operating model 1 to 7 describes the power transmission in half the cycle and the process of realizing soft switch. The operating model curves are symmetrical. In the next half cycle, the process is similar, and it will not be described further here.

\subsection{Switch loss calculation}

It can be known from the power transmission process of half the cycle that $S_{2}, S_{3}$ and $S_{5}, S_{8}$ realize ZVS turning on, $S_{1}$ and $S_{4}$ realize ZCS turning off. At the moment of $t=t_{4}, S_{5}$ and $S_{8}$ are not triggered, its current directional is transferred. During the transfer process, $S_{5}$ and $S_{8}$ are hard turn off, and $D_{6}$ and $D_{7}$ realize hard turning on. The half cycle power transmission loss can be obtained by the calculation of Formula (1):

$$
P_{\text {loss }}=2 \times \frac{1}{T}\left[\int_{t_{5}}^{t_{5}+t_{\text {off }}} i_{s 5}(t) \times V_{s 5}(t) \times f_{s w} d t+\int_{t_{5}}^{t_{5}+t_{o n}} i_{D 6}(t) \times V_{D 6}(t) \times f_{s w} d t\right]
$$

Where $T$ is switching period, $f_{s w}$ is switch frequency, $t_{\text {off }}$ is $S_{5}, S_{8}$ turn-off time, $t_{o n}$ is $D_{6}$, $D_{7}$ turn-on time. $i_{s 5}(t), V_{s 5}(t)$ are $S_{5}$ 's voltage and current expression formulas in $t_{5} \sim t_{5}+t_{\text {off }}$ 
period respectively, $i_{D \sigma}(t)$ and $V_{D \sigma}(t)$ are $D_{6}$ voltage and current expression formulas in $t_{5} \sim t_{5}+t_{\text {on }}$ period respectively. It can be know from topological structure symmetry that the entire cycle loss is twice $P_{\text {loss. }}$.

\section{Circuit parameters and controller design}

\subsection{Main circuit parameter calculations}

In order to achieve the design of DC/DC converter for PEMFC, each model source should keep constantly $7.5 \mathrm{~kW}$ output power. As the output voltage range of fuel cell is from $40 \mathrm{~V}$ to $75 \mathrm{~V}$ with big change scope, the choice of transformer turns ratio $\mathrm{n}$ is considered by the actual needs of high step-up ratio, as well as to consider the impact of turns ratio $\mathrm{n}$ on transmission loss. Therefore, available turns ratio should be selected to get lower switch loss and higher transmission efficiency. So lay down $n=7$, duty cycle $D=0.75$, switch frequency $f_{s}=10 \mathrm{kH}$.

According to Formula (2) transformer's leakage inductance is:

$$
L_{l k}=\frac{V_{o} \cdot(D-0.5)}{2 n \cdot I_{i n} \cdot f_{s}}
$$

From turns ratio $n$, leakage inductance $L_{l k}$ and $V_{o}$ determines the changing speed of leakage inductance current $i_{l k}$ is determined. And this value is fixed. The corresponding model duration can be calculated according to current slope. The specific results are obtained from (3) calculation:

$$
\left\{\begin{array}{l}
\Delta i_{l k}=0-i_{i n} \\
\frac{\Delta i_{l k}}{\Delta t_{12}}=\frac{d i_{l k}}{d t} \\
\frac{d i_{l k}}{d t}=-\frac{V_{o}}{n L_{l k}}
\end{array}\right.
$$

Where $\Delta t_{12}$ is the specific time of operating model $2, \Delta i_{l k}$ is the changing amount of leakage inductance current at that time period. Boost inductance $L$ ensures current's stable and continuous. In case of closed loop, the boost inductance can be obtained from Formula (4) ${ }^{[7]}$ is:

$$
L \geq \frac{V_{i n} \cdot(D-0.5)}{\Delta I_{i_{n}} \cdot f_{s}}
$$

Where $\Delta i_{\text {in }}$ is the ripple wave current through $L$.

\subsection{Power closed loop design}

Two polarization effects of activation polarization and ohm polarization are considered in this paper. The corresponding polarization curves were fitted by actual PEMFC parameters. In PSIM by using C-Block module the non-linear PEMFC external feature model was built. By testing the primary side power $P_{\text {in }}$ of current-fed DC/DC converter and compare it to 
$P_{r e f}$, and the difference $e_{r r}$ is input into PI controller, the output power was clipped to obtain duty cycle $d^{\prime}$ to adjust $t_{34}$ and $t_{67}$. According to current type DAB wave generating law, output duty ratio $d$ is used to adjust duty ratio of different switch tubes, eventually the output power is kept a stable value through controlling the size of transmission power. The closed loop system control frame figure is shown as Fig.4. In order to simulate big capacity $750 \mathrm{~V}$ DC bus, increase the magnitude of DC bus capacitance $C_{0}$ in PSIM. Meanwhile to ensure $7.5 \mathrm{~kW}$ constant power output, take $R_{0}$ as $75 \Omega$, so there's method to close loop control the output side power. As the power transmission efficiency of current type DAB, the difference between input and output sides is not so big, hence the output power $P_{\text {in }}$ of the non-linear power PEMFC is taken as the control objective. This similarity is only used for simulated analysis, in actual circuit, the output power is still used to conduct closed loop control.

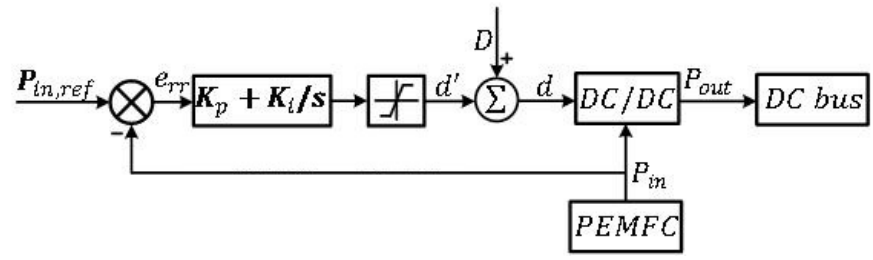

Fig. 4. Control block diagram of closed loop system.

\section{PSIM emulation}

The non-linear PEMFC module is built by using PSIM, and the main circuit of current-fed dual-active full-bridge DC/DC converter is shown as Fig.5. The main circuit parameters are set according to parameter calculation in section 4.1. By collecting the output voltage and current signals of PEMFC, it is fed back to the power closed-loop algorithm module for calculation, and the calculation results are input into the current type DAB modulation module to adjust the duty cycle of different switches according to the wave generation law of current type DAB.

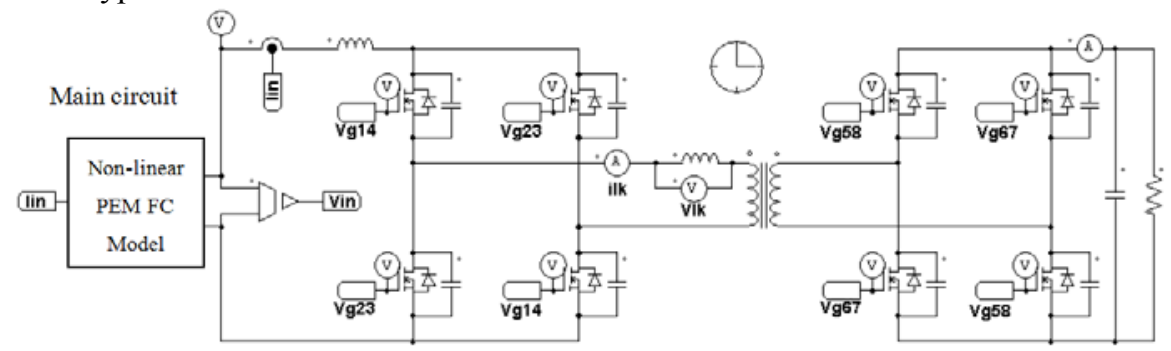

Fig. 5. PEMFC current-fed dual-active full-bridge DC/DC converter PSIM main circuit structure diagram.

Fig.6 shows the actual voltage and current oscillogram of non-linear PEMFC module. Under the effect of power closed loop, during activation polarization and ohm polarization, the output voltage is reduced with the current increasing, and eventually the voltage and current tend to be stabilized. 


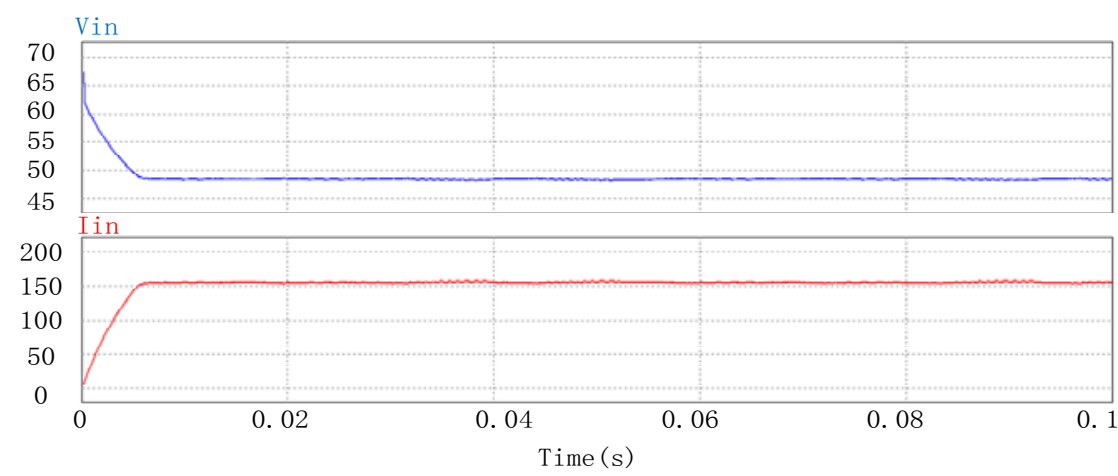

Fig. 6. PEMFC non-linear working waveform.

Fig.7 is the waveform of leakage inductance $L_{l \mathrm{k}}$ voltage and current. During the entire dynamic process, the voltage does not have obvious peak, and the current has always been appearing dynamic change. Within half of the period, the occurrence of $i_{l k}$ current peak makes diodes $D_{1}$ and $D_{4}$ stream conduct, and ensure $S_{1}$ and $S_{4}$ zero current shutdown.

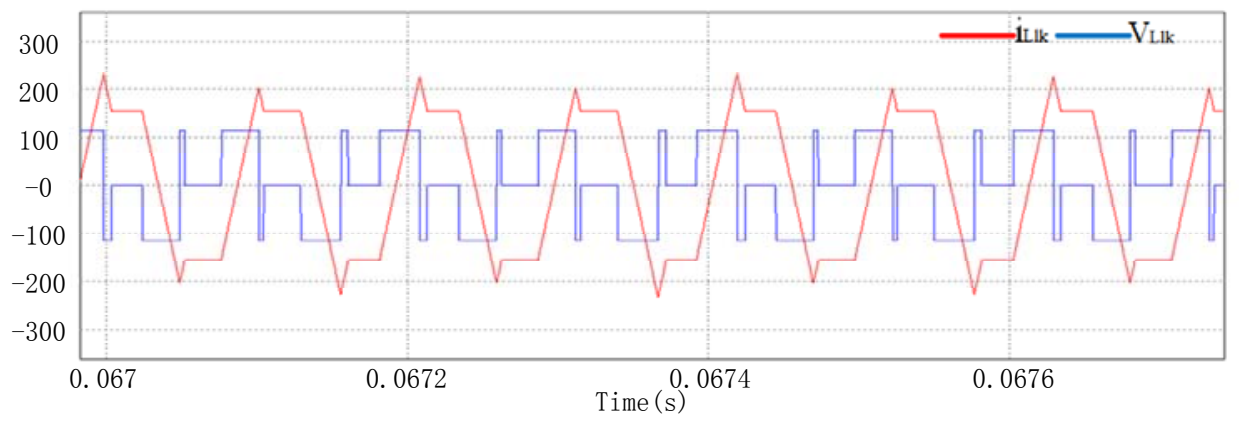

Fig. 7. Leakage inductance voltage and current waveform.

Fig.8 shows the realization process of soft switch, and only one switch tube with the same PWM control signals is displayed. For example, $S_{I}$ and $S_{4}$ have the same control signals, $S_{1}$ voltage and current waveform is only shown in the Fig.9. In one cycle, $S_{5}$ and $S_{6}$ are ZVS, and $S_{1}$ and $S_{2}$ are ZVS and ZCS . Similarly in the same cycle, switch tubes $S_{2}, S_{3}$ and $S_{6}, S_{7}$ staggering corresponding with the same bridge $S_{1}, S_{2}$ and $S_{5}, S_{8}$ can also correspondingly realize soft switch operating. 

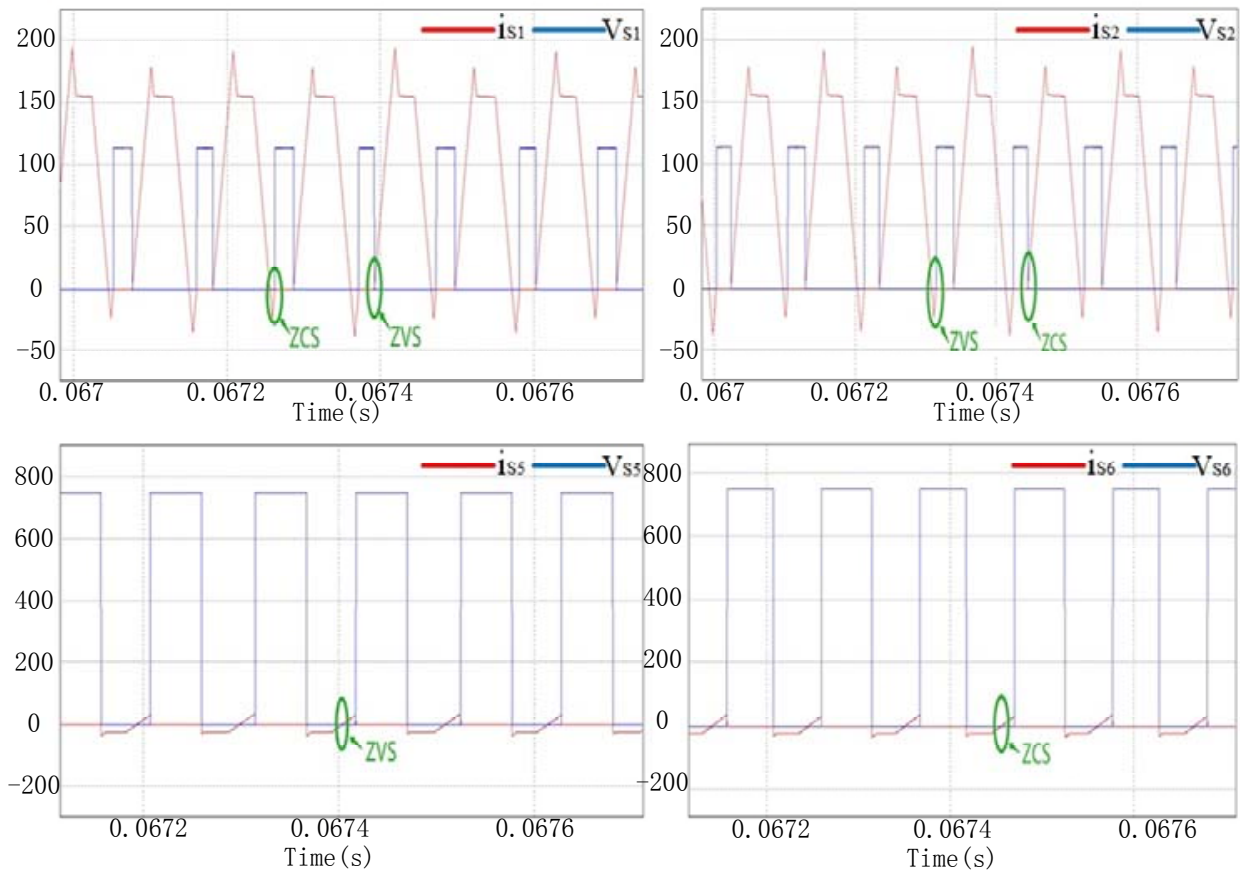

Fig. 8. Diagram of main waveforms of DC/DC converte.

In Fig.9, input side current ripple wave of current type DAB is shown. the size of the input current ripple wave covers $0.478 \%$ of the current average value. According to related documents conclusion, the high frequency current ripple wave $(1 \mathrm{kHz}, 10 \%$ ripple wave current amplitude) will not pose impact on PEMFC's service life ${ }^{[9]}$, thus satisfying the design requirement of the output current ripple wave.

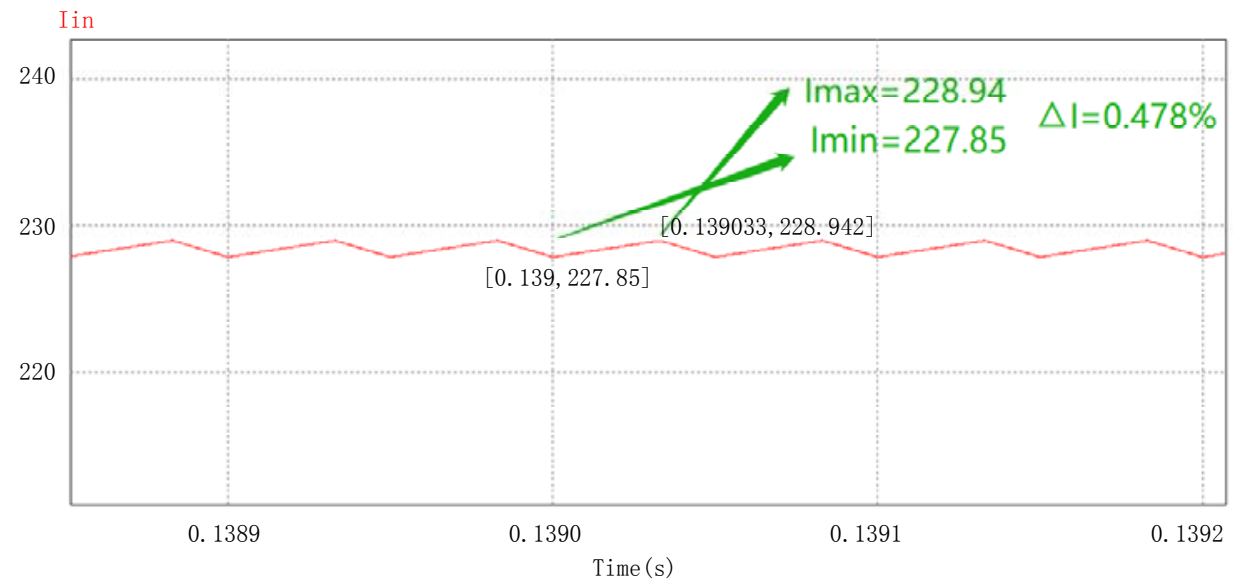

Fig. 9. DC/DC converter input current ripple expansion diagram.

Fig. 10 is the input/output power waveform diagram under closed loop control. $750 \mathrm{~V}$ DC power grid side needs constant $7.5 \mathrm{~kW}$ power, but during the time period of 0 to $7.5 \mathrm{~kW}$, the supply and demand is imbalanced for the output power of the non-linear PEMFC output power. Because the DC power grid is simulated by capacitor, the capacitor outputs $7.5 \mathrm{~kW}$ 
constant power to the resistor $R_{0}$, the capacitor voltage drops briefly, until the end of this transition process, the input and output power tends to be stable.

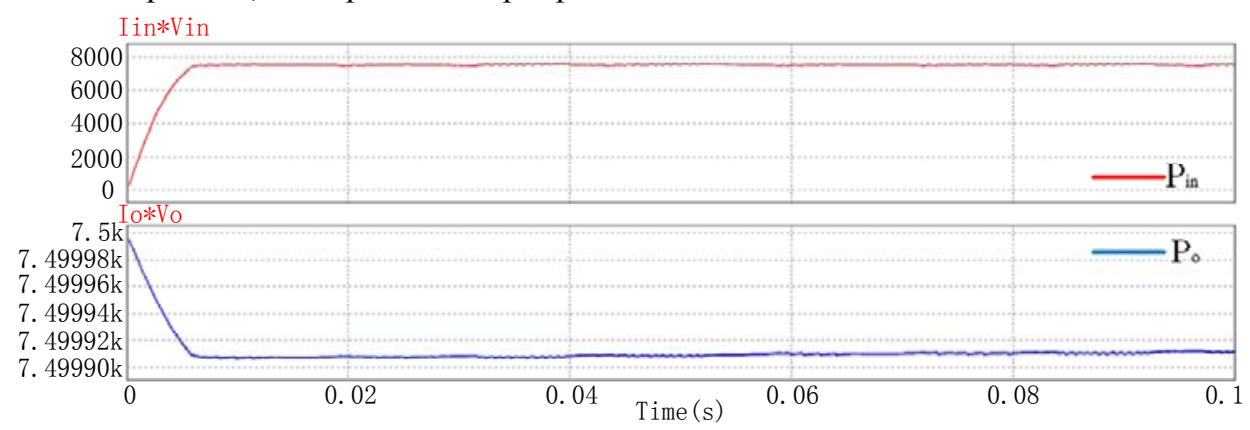

Fig. 10. DC/DC converter input and output power waveform.

In Fig.11, the expansion waveform of input and output power is shown, which is the average power of the input and output power in the displayed zone. The input average power is $7.52 \mathrm{~kW}$, output power is $7.47 \mathrm{~kW}$, the efficiency is $98.57 \%$.

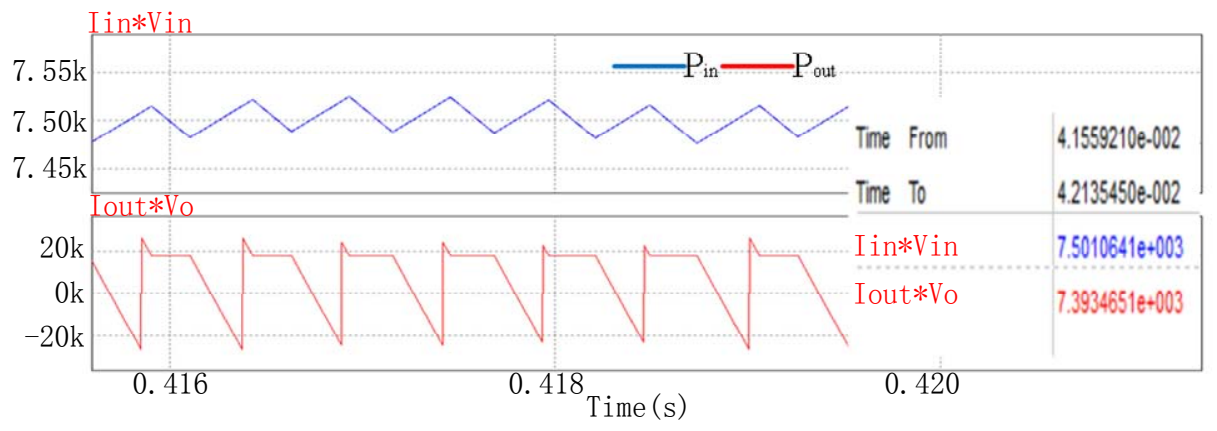

Fig. 11. DC/DC converter input and output power waveform expansion diagram.

\section{Conclusion}

In this paper, considering the nonlinear output characteristics of PEMFC and the high requirement of output current ripple, the current model DAB is applied to the constant power output of PEMFC to $750 \mathrm{~V}$ DC bus. Through theoretical analysis, power closed-loop design and simulation experiments, it is verified that the topology can achieve efficient and constant power transmission. In addition, the current fed dual active full bridge DC/DC converter achieves zero voltage on, zero current off of primary switches and zero voltage on of secondary switches, and $0.478 \%$ high frequency input current ripple will not affect the life of PEMFC, which meets the requirements of constant power transmission efficiency and current ripple of nonlinear power supply PEMFC.

\section{Acknowledgments}

This work was supported by State Grid Co., Ltd. Science and Technology Project: Research on Key Technologies of Proton Exchange Membrane Fuel Cell Connected to Power Grid Based on Rich Hydrogen Production (Project No. 5419-202020249A-0-0-00). 


\section{References}

1. H. LIU. Modeling and Simulation of PEMFC. North China Electric Power University (2012)

2. Y. Shi, R. Li, Y. Xue, et al. Optimized Operation of Current-Fed Dual Active Bridge DC-DC Converter for PV Applications. IEEE Transactions on Industrial Electronics, (2015), 62(11):1-1.

3. AK Rathore, U R, P. Analysis, Design, and Experimental Results of Novel Snubberless Bidirectional Naturally Clamped ZCS/ZVS Current-Fed Half-Bridge DC/DC Converter for Fuel Cell Vehicles. IEEE Transactions on Industrial Electronics, (2015), 60(10):4482-4491.

4. X Pan, Z Xin, Z Peng, A Ghoshal, AK Rathore. Hybrid Modulation Based Bidirectional Electrolytic Capacitor-less Three-phase Inverter for Fuel Cell Vehicles: Analysis, Design, and Experimental Results. IEEE Transactions on Power Electronics, (2017):1-1.

5. D Sha, G Xu. High Efficiency Current-Fed Dual Active Bridge DC-DC Converter with ZVS Achievement Throughout Full Range of Load Using Optimized Switching Patterns. IEEE Transactions on Power Electronics, (2018):1-1.

6. D Sha, X Wang, K Liu, C Chen. A Current-Fed Dual-Active-Bridge DC-DC Converter Using Extended Duty Cycle Control and Magnetic-Integrated Inductors With Optimized Voltage Mismatching Control. IEEE Transactions on Power Electronics, (2018).

7. P Xuewei, Rathore, A.K.. Novel Bidirectional Snubberless Naturally Commutated Soft-Switching Current-Fed Full-Bridge Isolated DC/DC Converter for Fuel Cell Vehicles. IEEE Transactions on Industrial Electronics, (2014), 61(5):2307-2315.

8. J. He, C. Sun. Modeling and simulation analysis of PEMFC.Journal of Central South University(natural science),(2010),41(02):566-571.

9. B Wahdame, L Girardot, D Hissel, et al. Impact of power converter current ripple on the durability of a fuel cell stack. IEEE International Symposium on Industrial Electronics. IEEE, (2008). 